\title{
Associations involving delays (particularly long delays) between certain weather parameters and geomagnetic activity
}

\author{
G. G. Bowman and I. K. Mortimer \\ Department of Physics, The University of Queensland, Brisbane 4072, Australia \\ (Received November 15, 2000; Revised May 7, 2001; Accepted September 3, 2001)
}

\begin{abstract}
Four sunspot-minimum periods (1963-1966, 1971-1977, 1983-1987 and 1992-1997) have been examined for the results which are presented. Using several different weather parameters, tropospheric gravity waves, enhanced cold fronts and two rainfall data sets in Eastern Australia, associations at reasonably high levels of significance have been found with enhanced geomagnetic activity (EGA). Statistically this EGA involved either short delays of several days or long delays of about 20 days. The geomagnetic parameters used were (a) the AE index (b) the hourly $\mathrm{H}$ component for a number of stations and (c) the daily $K_{P}$-sum value. The $K_{P}$-sum analyses have shown that the EGA associated with the delays form part of four or five cycles of recurrent geomagnetic activity for 27-day periodicities. Furthermore statistically two recurrent cycles are found to exist concurrently, one apparently related to the short delays and the other to the long delays. Periodicities of 13.5 days are created because the two sets are displaced from each other by approximately this interval. A brief reference is made to the 13.5 periodicity known to exist for geomagnetic activity and the evidence in the literature for active regions on the sun to be displaced by 180 degrees of solar longitude.
\end{abstract}

\section{Introduction}

There have been many papers relating to sun-weather relationships over the last century. Projects in recent times have examined for example temperature changes (Bucha and Bucha, 1998), cloud cover related to cosmic ray flux (Svensmark and Friis-Christensen, 1997) and electrofreezing (Tinsley, 1996). One particular type of investigation, which dates back some time, is concerned with weather changes delayed a few days after enhanced geomagnetic activity (EGA) (Roberts and Olson, 1973; Bucha and Bucha, 1998). An early result by Duell and Duell (1948) which is partially reproduced by figure 1 of Bowman and Shrestha (1998) is representative of this relationship. The literature on short-term relationships with EGA is discussed in some detail by Bowman and Shrestha (1998). Recent papers which cover four sunspot-minimum periods (1963-1966, 1971-1977, 1983-1987 and 1992-1997) report on tropospheric gravity waves (also known as ground-level atmospheric gravity waves-GL-AGWs) recorded at Brisbane, Australia, (Bowman and Shrestha, 1998; Bowman, 1972, 2000). These give details of associations which these events apparently have with EGA a few days earlier. These will be called type 1 delays. Results relating to these type 1 delays will be presented here. However the analyses which have been performed will emphasize the existence of long delays ranging from 15 to 20 days which are found associated with GL-AGWs as well as some other weather parameters. Although the results for the initial analyses of this phenomenon (long delays) seemed to be statistically significant, mecha-

Copy right(c) The Society of Geomagnetism and Earth, Planetary and Space Sciences (SGEPSS); The Seismological Society of Japan; The Volcanological Society of Japan; The Geodetic Society of Japan; The Japanese Society for Planetary Sciences. nisms which might be involved were difficult to imagine. These long delays will be called type 2 delays.

The weather parameters to be considered will be GLAGWs, certain cold-front events, and some events associated with rainfall areas in Eastern Australia. In Eastern Australia the local time (LT) is 10 hours ahead of universal time $(\mathrm{UT})$ (i.e. $\mathrm{LT}=\mathrm{UT}+10 \mathrm{~h}$ ). Table 1 lists the corrected geomagnetic coordinates for the stations which will be mentioned here, in order of descending longitude.

\section{Methods of Analysis}

The superposed-epoch method has been used throughout, the period covered in each analysis being extensive with error bars and standard-deviation displacements available in the limited sections of the results which will be presented. For example analyses which use hourly values cover the centre hour (arbitrarily chosen as $0000 \mathrm{UT}$ ) \pm 30 days (i.e. 1441 hours). The details of these methods have been given in the earlier papers (Bowman and Shrestha, 1998; Bowman, 2000). The geomagnetic parameters used are (a) AE indices, (b) the $\mathrm{H}$ components at a range of stations and (c) $K_{P}$-sum values. For some results spectral analyses have also been performed. Because of annual and sunspot-cycle fluctuations (see e.g. Taylor et al., 1996) the $K_{P}$-sum values have been normalized by dividing successive 61 day intervals during the year by the average value for each interval. For normalization the results obtained were somewhat between than when it was not used. Furthermore to modify rapid fluctuations over short periods 5-day running means have been used on these $K_{P}$-sum values. 


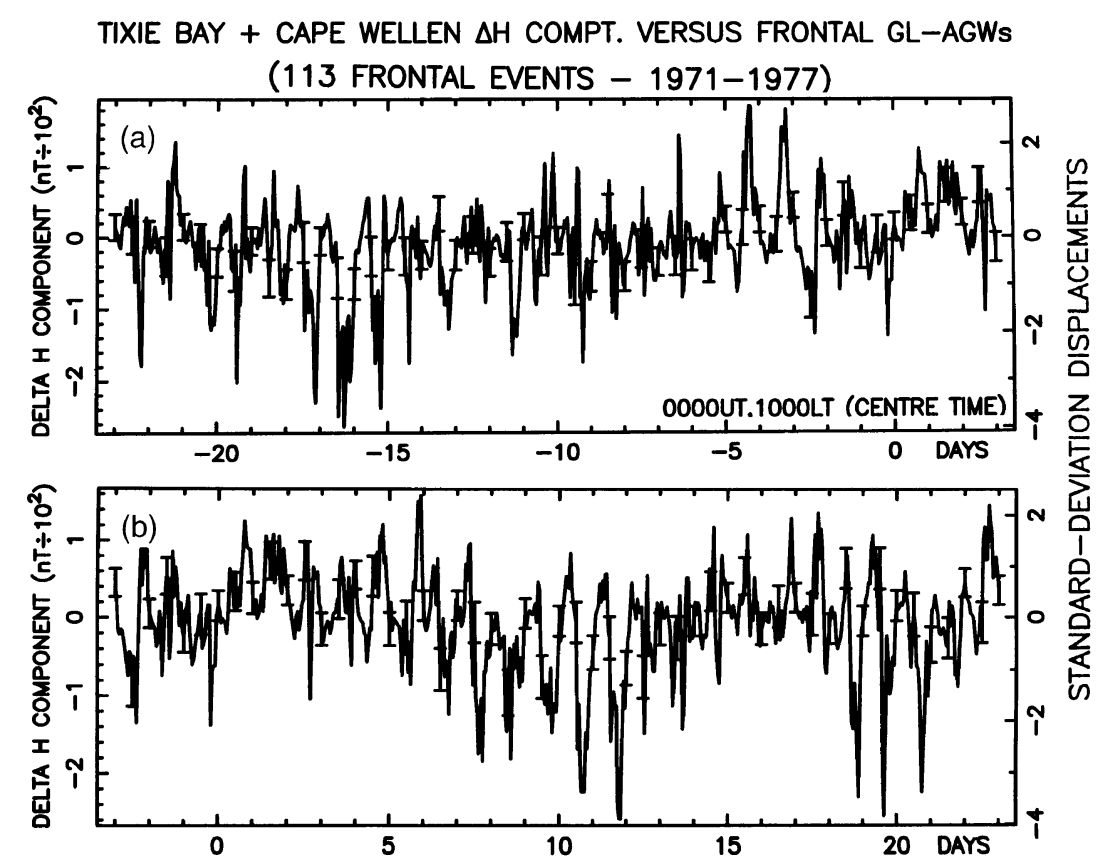

Fig. 1. (a) and (b) $\Delta H$ components for Tixie Bay and Cape Wellen relative to 113 frontal GL-AGWs (1971-1977) from -23 to +23 days.

Table 1. Corrected geomagnetic coordinates.

\begin{tabular}{lrr}
\hline \multicolumn{1}{c}{ Stations } & \multicolumn{1}{c}{ Lat. } & Long. \\
\hline College & 64.6 & 259.4 \\
Cape Wellen & 62.5 & 242.6 \\
Brisbane & -36.6 & 225.6 \\
Tixie Bay & 65.5 & 194.4 \\
Geraldton & -41.0 & 183.4 \\
Dixon & 67.9 & 154.0 \\
Murmansk & 64.6 & 114.1 \\
Leirvogur & 66.8 & 69.5 \\
\hline
\end{tabular}

\section{The Detection of Long Delays}

The GL-AGWs considered here will be those associated with cold fronts which pass through Brisbane a day or two after the GL-AGWs are recorded (called frontal GL-AGWs). Figure 1 shows analysis results involving hourly $\Delta H$ components at Tixie Bay and Cape Wellen relative to the frontal GL-AGWs for the period 1971 to 1977 . Besides the type 1 delays around 4 days there are also significant displacements centred on -16 days (Fig. 1(a)) and again at +12 days (Fig. 1(b)), an interval of 28 days. It has been found for the extensive superposed-epoch analyses of this kind that the final displacements are distributed normally (see e.g. Bowman and Mortimer, 2000). Consequently on Fig. 1 the probability of displacements around $4 \sigma$ being random is 0.006 percent (Croxton and Cowden, 1955), and these displacements of significance are repeated on consecutive days. The significance of these long delays is reinforced by the fact that similar $\Delta H$-component analyses at four other stations (College, Dixon, Murmansk and Leirvogur), distributed around the world, produced similar results (see
Fig. 2(b) for Murmansk). The significant displacements near -17 days on Fig. 2(a) are produced by an analysis using hourly AE indices relative to frontal GL-AGWs for the period 1971-1975. It should be noted that the occurrence times for EGA are concentrated around the local time of 0500, which is consistent with the results for type 1 delays for GLAGWs (see table 3 of Bowman and Shrestha, 1998).

This AE analysis also reveals a 28-day periodicity for the long delays (not shown) with enhanced displacements recorded at +11 days. As will be mentioned later, the type 1 delays are weak for the 1971-1977 period and although shown in Fig. 1(a), are not recorded in Fig. 2(a).

\section{Analyses Using $K_{P}$-Sum Values}

The significant displacements at 27 or 28 day intervals in Section 3 suggest that they might be part of the phenomenon of recurrent geomagnetic activity related to the rotation of the sun. Section 3 used hourly values, the analyses covering periods from -30 to +30 days relative to the centre hour. The daily $K_{P}$-sum values have been used to examine the extent of any recurrent tendency suggested by the use of hourly values. Here the coverage was centre day \pm 365 days (i.e. 731 days).

\subsection{Tropospheric gravity waves}

Figures 3 and 4 show the results for the $K_{P}$-sum analysis for each of the four periods for the GL-AGWs. There is evidence of both type 1 and type 2 delays each being part of recurrent periodicities of around 27 days. The peaks relating to the type 2 delays are dominant compared with those for the type 1 delays. Because of this and the need to use running means for results to be meaningful, the suppression of the type 1 events may be partly explained. The type 2 oscillations can be clearly recognized in Figs. 3(a) and 3(b) (for 1963-1966 and 1971-1977 respectively), while there is some evidence of type 1 delays in Figs. 4(a) and 4(b) 


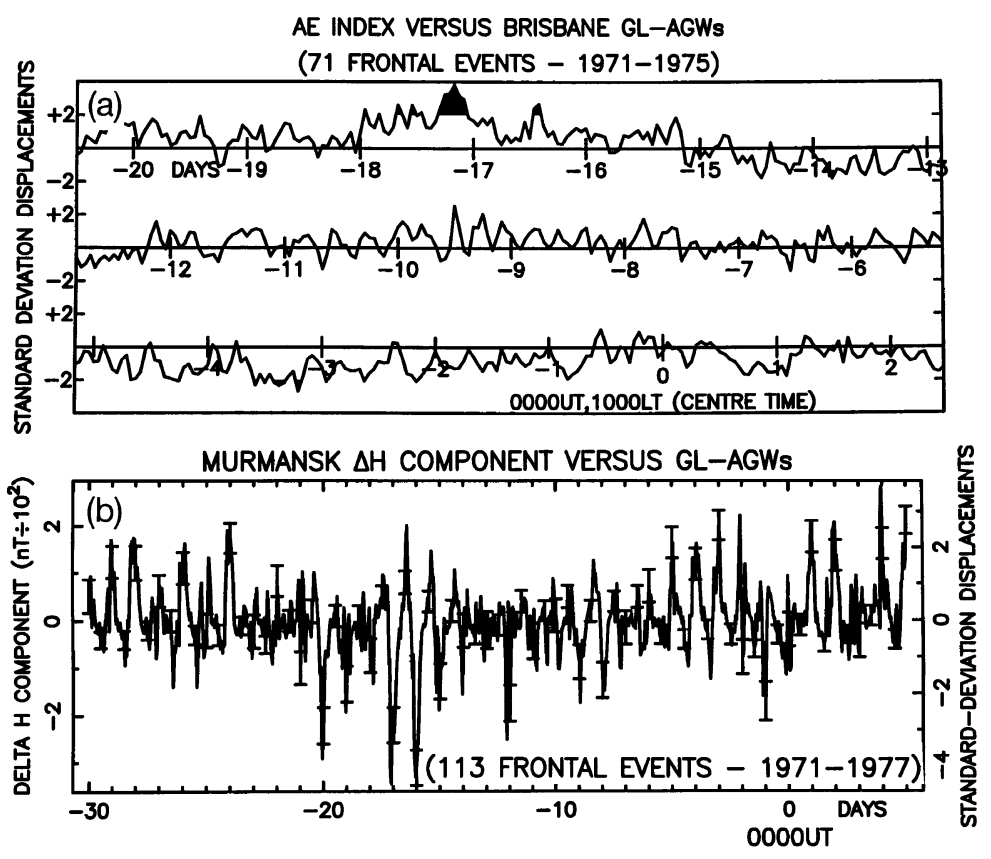

Fig. 2. (a) AE index and (b) Murmansk $\Delta H$ component relative to the tropospheric gravity waves used for Fig. 1.

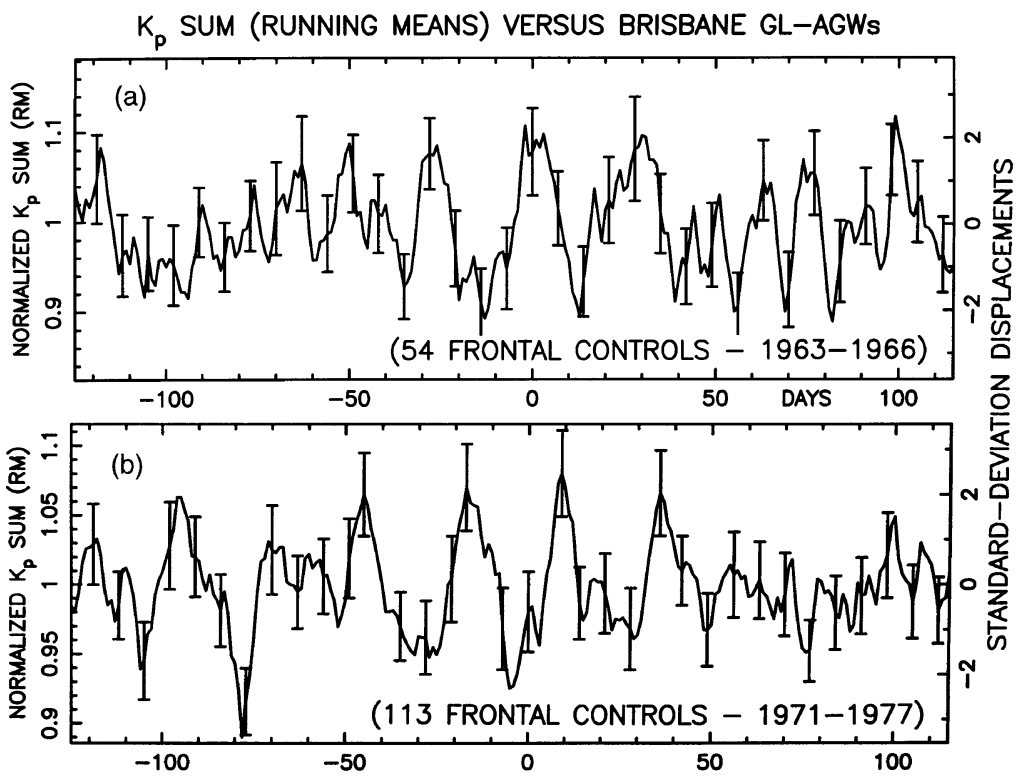

Fig. 3. $K_{P}$-sum analyses using frontal GL-AGWs for (a) 1963-1966 and (b) 1971-1977.

(for 1983-1987 and 1992-1997 respectively). Judging from the displacements of the other maxima it is estimated that the delay for the broad maximum type 2 delay for 19631966 is 24 days. Since the delays for 1983-1987 was 21 days and 21 or 22 days for 1992-1997 it was decided for the increased event numbers to combine these results into a single analysis. Consistent with the earlier results (Figs. 1 and 2) the type 2 delay for 1971-1977 is 17 days (Fig. 3(b)). Although other results will be presented and discussed later it should be noted here that spectral analyses have been performed for the four distributions in Figs. 3 and 4. These results (not shown) reveal periodicities around 27 and 14 days, as expected from for example the type 1 and type 2 peaks in Fig. 4(b). However the 14 day periodicity for the 1971-1977 period is not prominant, consistent with the earlier comment of the weakness of the type 1 events for this period.

The central section (centre day \pm about 100 days) of the results for the combined analysis (1963-1966; 1983-1987 and 1992-1997) is shown in Fig. 5(a). A dominant feature is a series of maxima spaced about 27 days with one maxima around -20 days. The standard-deviation displacements oscillate between $-2 \sigma$ and $+2 \sigma$ with one displacement of $+3 \sigma$. Oscillations which relate to the type 1 delays seem 


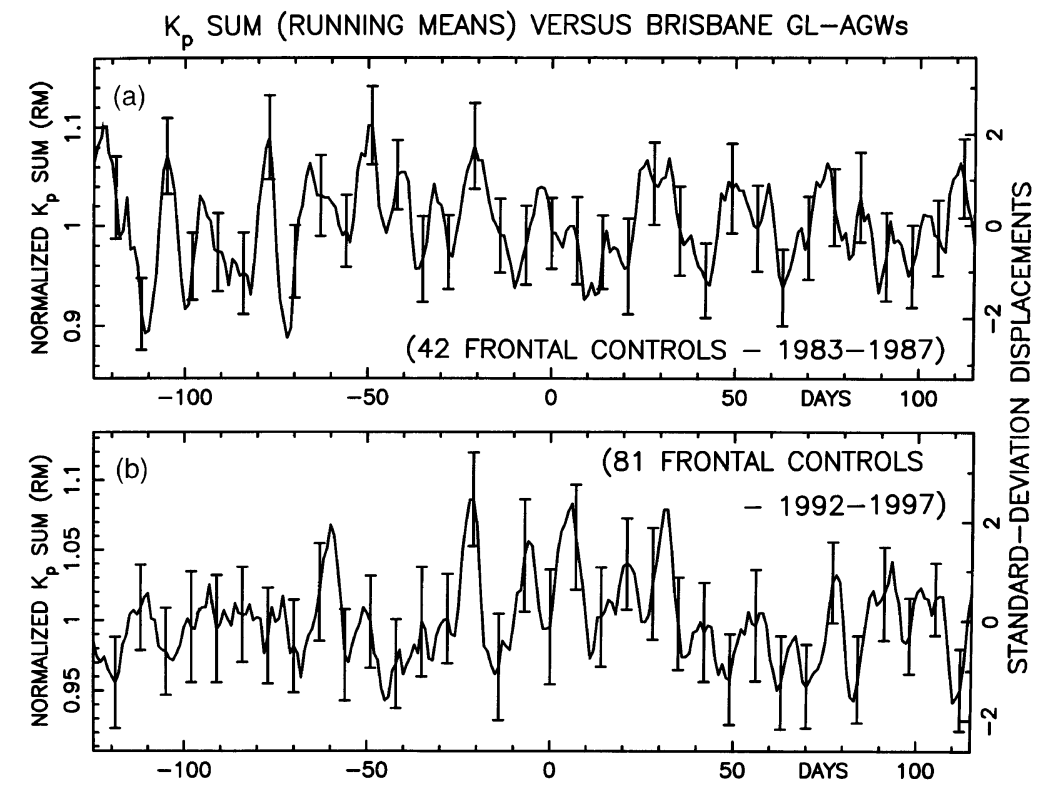

Fig. 4. $K_{P}$-sum analyses using frontal GL-AGWs for (a) 1983-1987 and (b) 1992-1997.

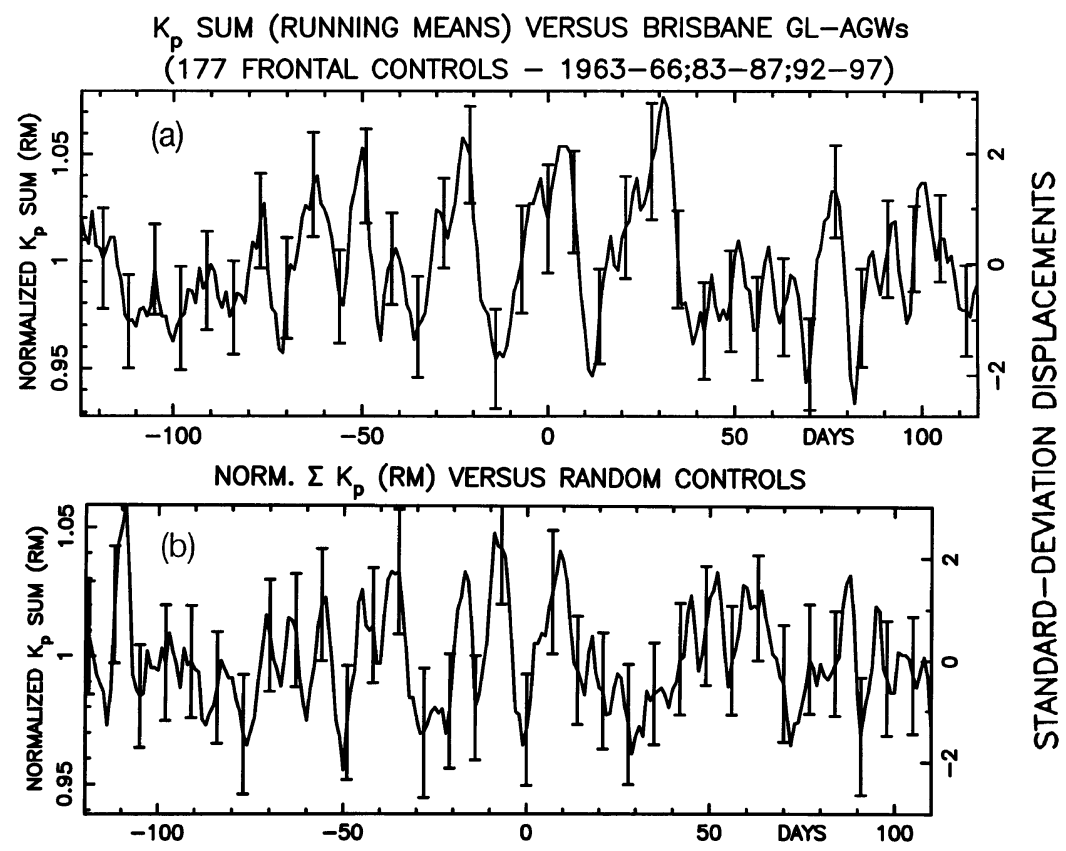

Fig. 5. $K_{P}$-sum analyses (a) for combined frontal GL-AGWs for 1963-1966, 1983-1987 and 1992-1997, and (b) same number of random controls.

to be masked by the more dominant events which relate to the type 2 delays. However the spectral analysis for the Fig. 5(a) distribution reveals clearly peaks around 27 and 14 days (Fig. 6(a)). As a further test of the significance of these results for GL-AGWs a similar number of random dates from each period used (totalling 177) have been used as controls. These give a distribution shown in Fig. 5(b) which is quite unlike that in Fig. 5(a). The spectral analysis for this distribution is shown in Fig. 6(b) where there is no evidence of dominant 27 or 14 day periodicities.

\subsection{Enhanced cold-front events}

As might be expected analyses for particular cold-front events produced associations (not shown) with EGA which were similar to those found for GL-AGWs, recorded a day or two earlier (Fig. 5(a)). However it was surprising and interesting to also find associated with EGA, although not as well defined, the other cold-front passages at Brisbane, over the four sunspot-minimum periods which did not have associated GL-AGWs. There were 370 such events. This result suggested an analysis of the 667 fronts which extended at least as far north as Geraldton in Western Australia, some 

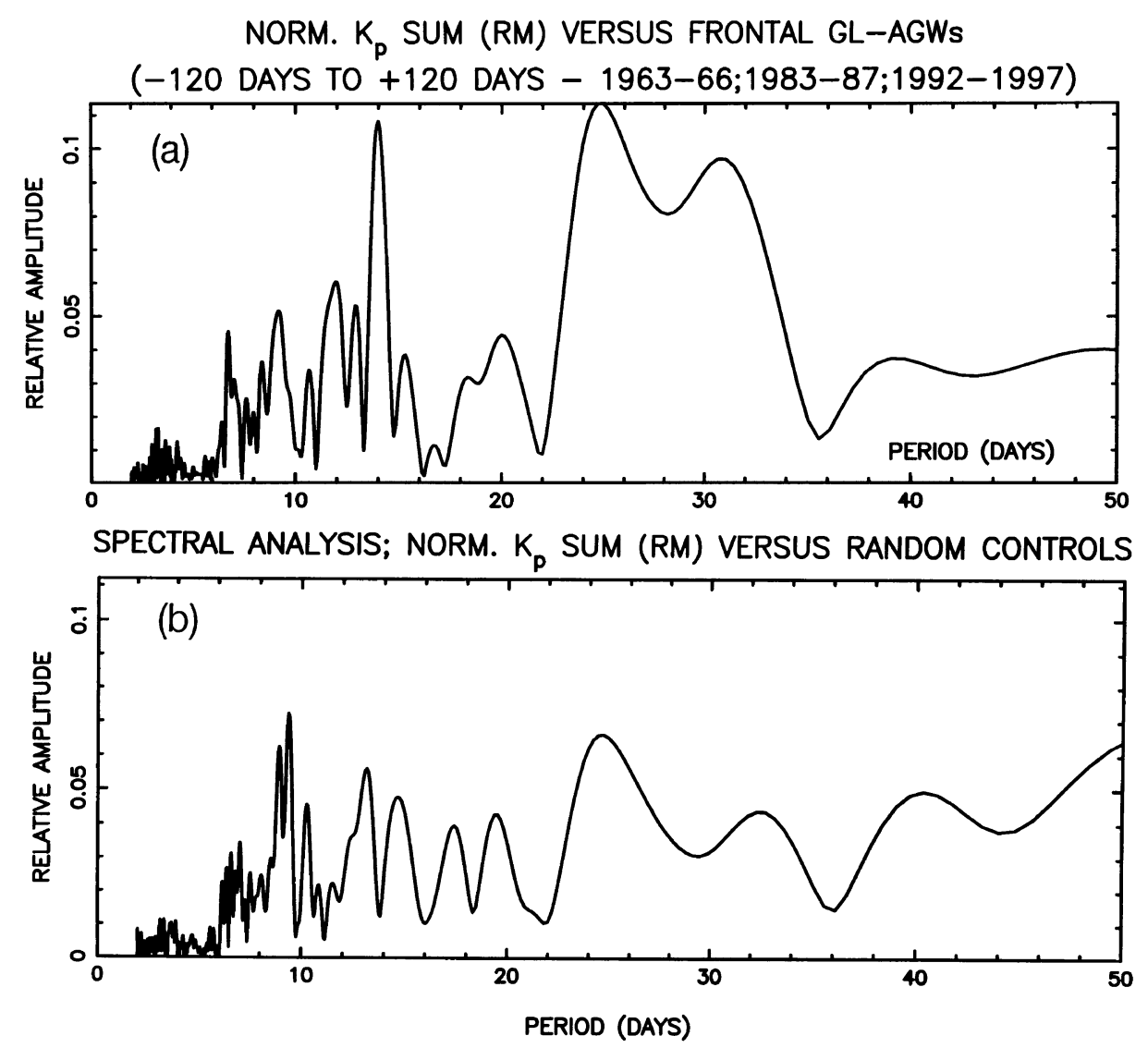

Fig. 6. Spectral analyses for $K_{P}$-sum distributions for (a) Fig. 5(a) and (b) Fig. 5(b).

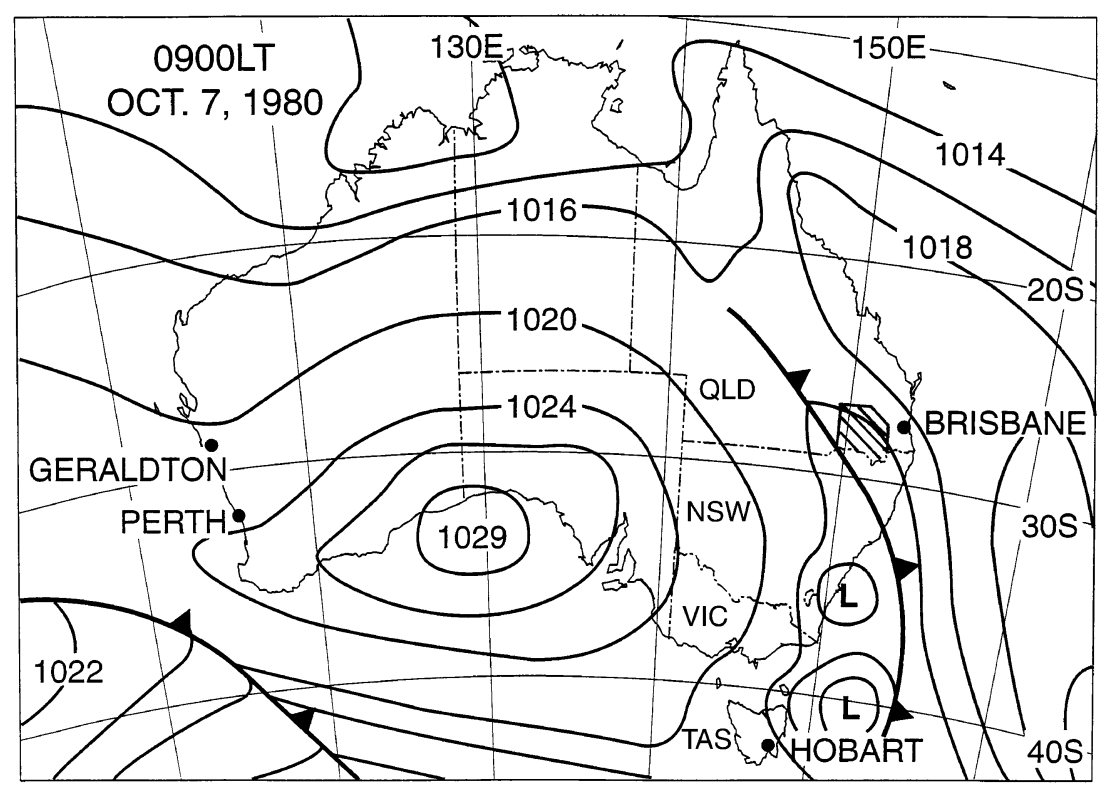

Fig. 7. Map of Australia showing locations of Brisbane, Geraldton and Darling Downs area.

$3700 \mathrm{~km}$ west of Brisbane. Geraldton's location is indicated in Fig. 7. Although cold fronts move across the Australian continent from west to east, a superposed-epoch analysis has shown that apparently the $3700 \mathrm{~km}$ separation is enough for the Brisbane and Geraldton events to be independent. As an analysis using the Geraldton fronts by themselves yielded results with EGA similar to those found for the Brisbane fronts not associated with GL-AGWs, these two event sets have been combined into one analysis involving $K_{P}$-sum values for the advantage of the increased event numbers. 


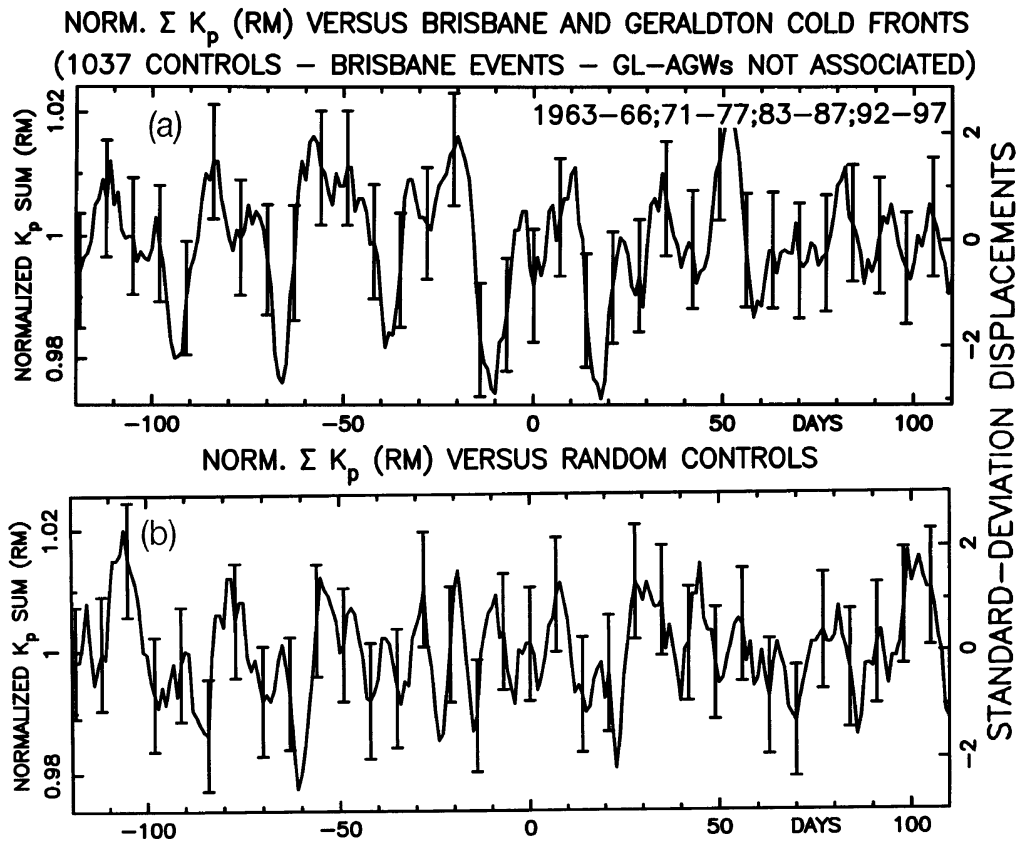

Fig. 8. $K_{P}$-sum analyses for (a) Geraldton and Brisbane (no GL-AGWs) cold fronts and (b) same number of random controls.

NORMALIZED $K_{p}$ SUM (RM) VERSUS BRISBANE AND GERALDTON COLD FRONTS
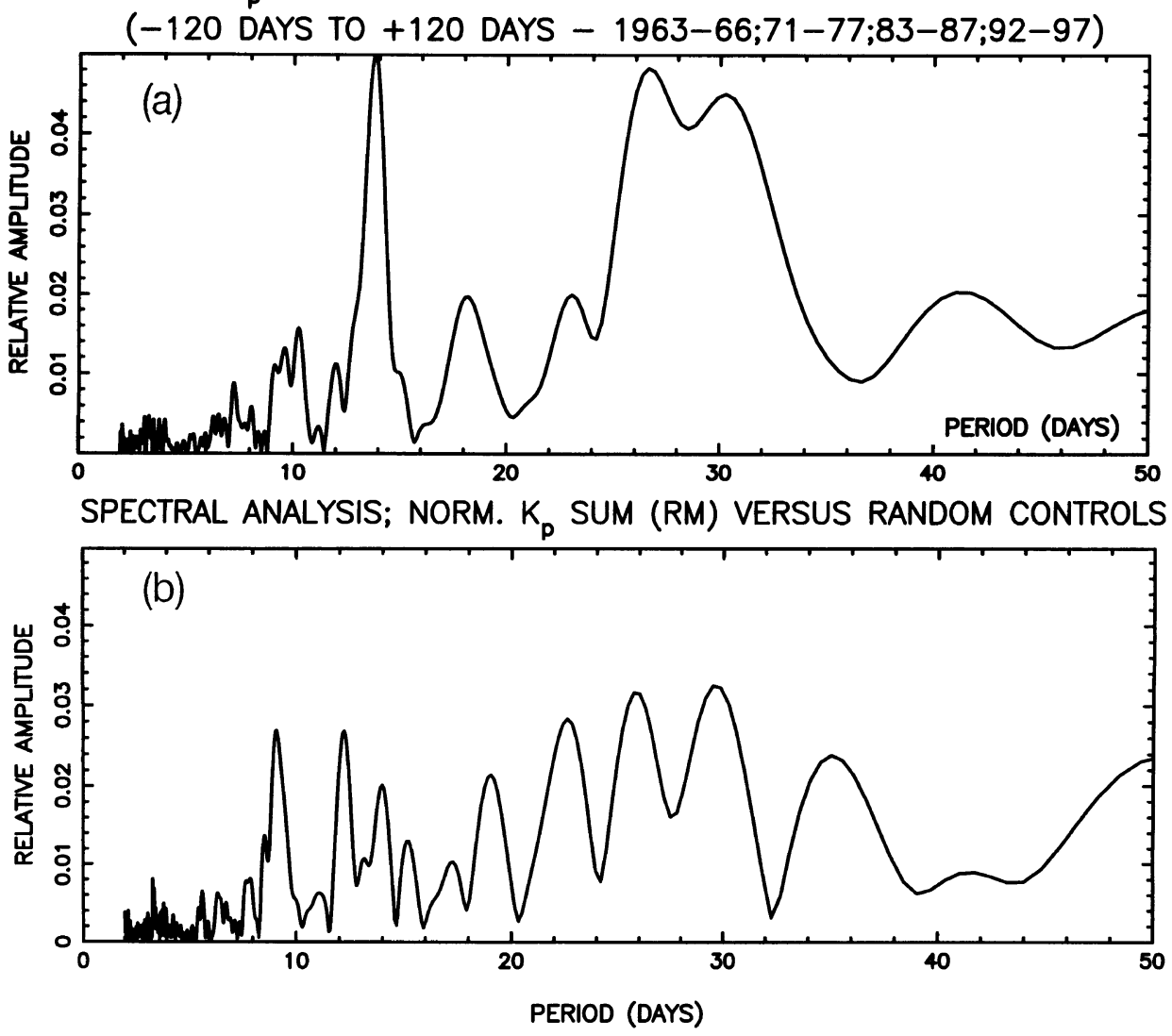

Fig. 9. Spectral analyses for $K_{P}$-sum distributions for (a) Fig. 8(a) and (b) Fig. 8(b). 
Table 2. Brisbane and Geraldton cold fronts (27-Day periodicities-Fig. 8(a)).

\begin{tabular}{|c|c|c|c|c|c|}
\hline \multicolumn{4}{|c|}{ Maxima } & \multirow{2}{*}{\multicolumn{2}{|c|}{$\begin{array}{l}\text { Minima } \\
\text { Series } 3\end{array}$}} \\
\hline \multicolumn{2}{|c|}{ Series 1} & \multicolumn{2}{|c|}{ Series 2} & & \\
\hline Days & $\sigma$ & Days & $\sigma$ & Days & $\sigma$ \\
\hline-111 & (1.4) & -75 & $(0.7)$ & -94 & $(-2.2)$ \\
\hline-84 & (1.5) & -49 & (1.3) & -66 & $(-2.7)$ \\
\hline-58 & (2.0) & -20 & (2.0) & -39 & $(-2.0)$ \\
\hline-33 & (1.2) & +11 & (1.4) & -10 & $(-2.9)$ \\
\hline-2 & (0.6) & +34 & (1.3) & +18 & $(-3.0)$ \\
\hline+20 & - & & & & \\
\hline+52 & (2.4) & & & & \\
\hline \multicolumn{2}{|c|}{6 cycles } & \multicolumn{2}{|c|}{4 cycles } & \multicolumn{2}{|c|}{4 cycles } \\
\hline Average & 27.1 days & Average & 27.2 days & Average & 28.0 days \\
\hline
\end{tabular}

The result for this combined analysis is shown in Fig. 8(a) and indicates the existence of two recurrent cycles with a separation of about 14 days. In addition five consecutive minima are well defined. Table 2 lists the details (location in days and standard-deviation displacements) of two maxima and one minima series. It is probably significant that quite independent of any GL-AGWs, Fig. 8(a) clearly shows appropriate values for the type 1 and type 2 delays. The large number of controls would have assisted the obtaining of meaningful results. The spectral analysis for this distribution which is shown in Fig. 9(a) is not unlike Fig. 6(a). A distribution obtained by using 1037 random controls is shown in Fig. 8(b) and the relevant spectral analysis in Fig. 9(b). There are no periodicities of interest in these two figures. The two recurrent series listed in Table 2 are confirmed with the well-defined 14 day periodicity indicative of the separation between these series.

\subsection{Some other weather systems and geomagnetic ac- tivity}

Since frontal GL-AGWs are associated with EGA (Bowman, 2000 and Fig. 5(a)), and independent of GLAGWs, enhanced cold fronts are found directly associated (Fig. 8(a)) it might be expected that certain aspects of rainfall may be found to also be associated. This subsection will report on two investigations into weather systems involving rainfall in Eastern Australia. All four sunspot-minimum periods were examined. The relevance of the results were variable with some revealing significant associations with EGA while for others interpretation of the results was more difficult. The best results were found for the 1971-1977 period where as Fig. 3(b) shows the 27-day periodicity associated with the type 2 delay is dominant. However significant results in other years will also be mentioned.

Associations of EGA with weather have been assessed in terms of rainfall areas in south-eastern Australia. These areas incorporate the states Tasmania (Tas), Victoria (Vic) and New South Wales (NSW) and are illustrated in Fig. 7. A classification of small was used if rainfall areas were $\geq 5$ but $<45$ percent of the total area of the three states; medium if $\geq 45$ but $<65$, large for areas $\geq 65$ percent and very large for rain areas extending into Queensland (Qld). Rainfall ar-
Table 3. Darling Downs rainfall levels.

\begin{tabular}{ccc}
\hline level & average mm & events 1971-1977 \\
\hline High & $\geq 10$ & 125 \\
Medium 1 & $\geq 4<10$ & 187 \\
Medium 2 & $\geq 2.5<4$ & 107 \\
Low 1 & $\geq 1.5<2.5$ & 102 \\
Low 2 & $\geq 0.5<1.5$ & 219 \\
Low 3 & $\geq 0.1<0.5$ & 307 \\
\hline
\end{tabular}

eas for 24 hours from 0900 (LT) on the event day were assessed from daily weather charts. Thus the event day interval (0900 to 0900) applies closely to that for the geomagnetic activity used in the analyses which is expressed in universal time. The $\Delta H$ components for Tixie Bay, Cape Wellen and College were examined relative to events representing each of these area classes. The small and medium levels showed little or no evidence of types 1(a) or 1(b) associations although the medium-area analysis indicated some support for the type 2 delays. The best results were obtained for the analysis involving the large areas. The results are shown in Figs. 10(a) and 10(b) for the 1971-1977 period. Here both the short and the long type delays can be recognized with many displacements exceeding $3.5 \sigma$. The displacement near -15 days is consistent with the results presented in Figs. 1, 2 and 3(b). There is however an additional 27-day range which extends from approximately -20 days to +7 days. A similar analysis for the 1983-1987 period and very large areas produced displacements around $5 \sigma$ at positions -19 and +7 days while the 1963-1966 period for medium levels had significant displacements around $4 \sigma$ at -15 and +13 days.

Another approach was to consider average daily rainfall levels for 9 or 10 stations in a uniform area of Queensland (Qld) called the Darling Downs. These levels were obtained from daily publications of rainfall for Queensland locations. They were used as controls in analyses involving the $\Sigma K_{P}$ parameter. This area of $70,000 \mathrm{~km}^{2}$ which is illustrated by 
TIXIE BAY + CAPE WELLEN $\triangle H$ COMPT. VERSUS LARGE RAINFALL AREAS
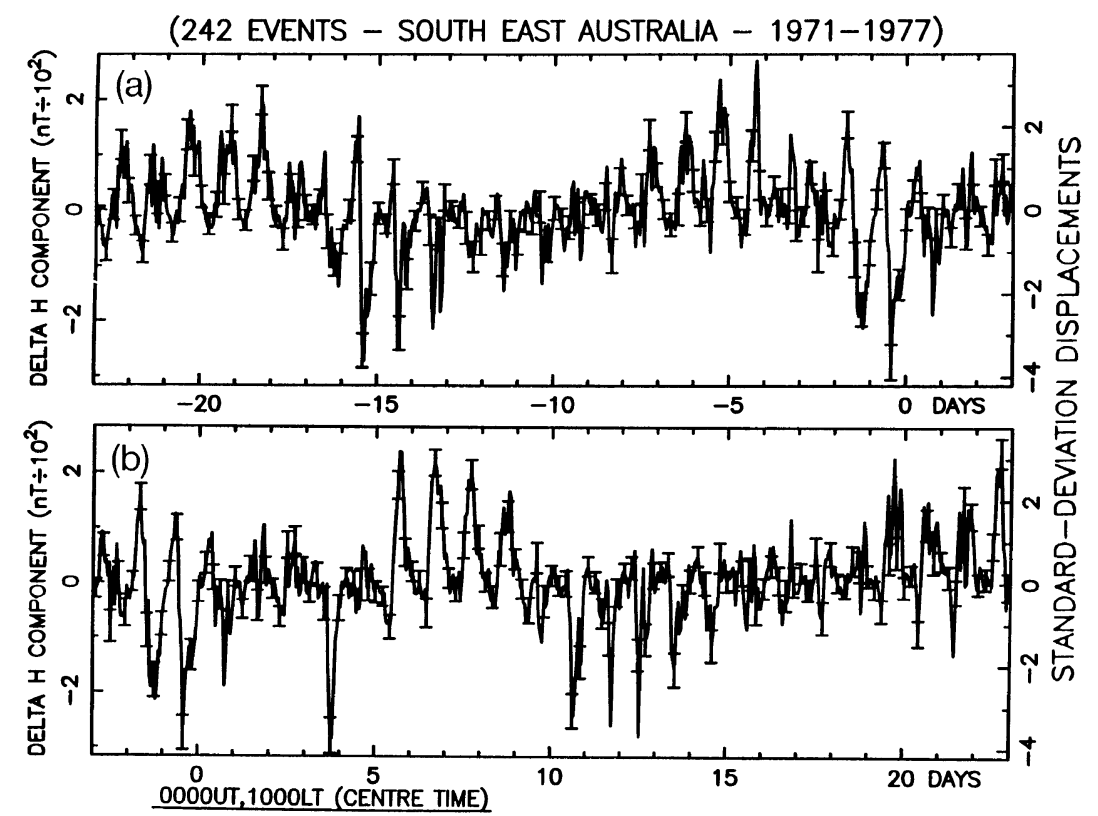

Fig. 10. (a) and (b) $\Delta H$ components for Tixie Bay and Cape Wellen relative to large rainfall areas from -23 to +23 days.
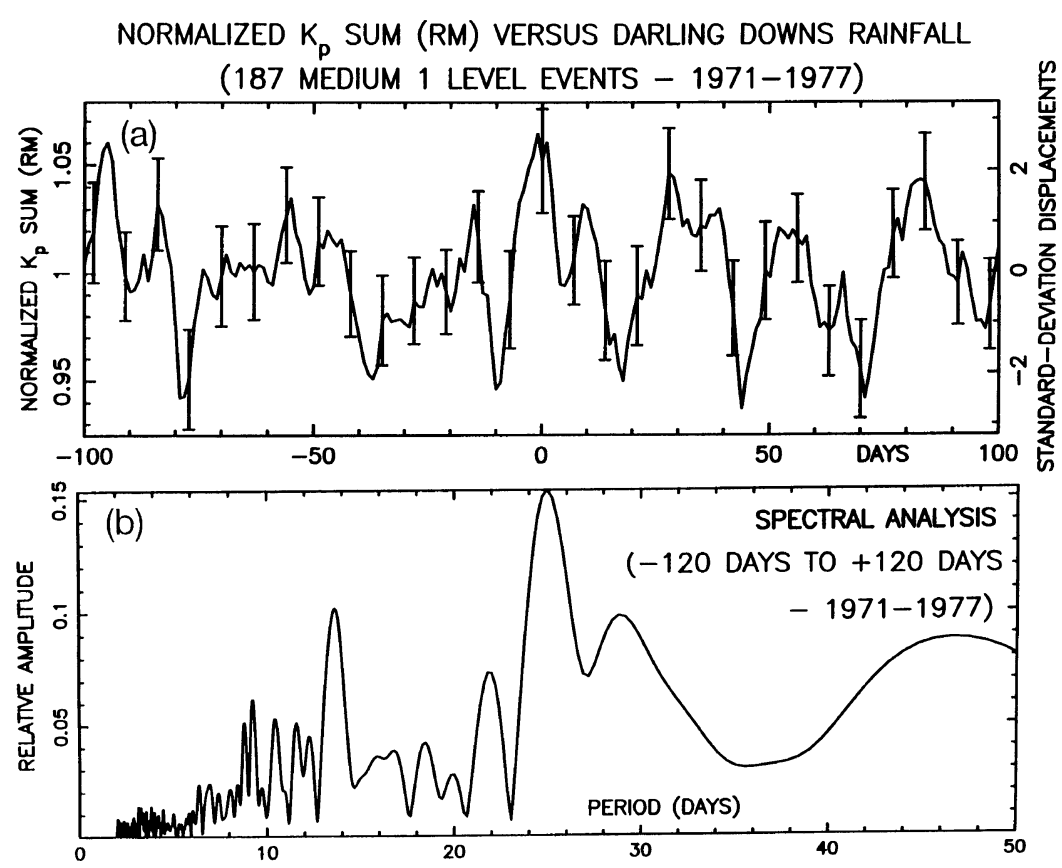

Fig. 11. (a) $K_{P}$ sum relative to Darling Downs medium rainfall levels and (b) spectral analysis of Fig. 11(a) distribution.

the shaded area of Fig. 7 has its centre located approximately $200 \mathrm{~km}$ west of Brisbane. The subdivision into the various rainfall ranges used and the occurrence days for 1971-1977 is shown in Table 3. The analyses for the medium 1 and 2 controls gave results consistent with those found earlier (Subsections 4.1 and 4.2).

Figure 11(a) shows the medium 1 results with the relevant spectral-analysis plot in Fig. 11(b). Similar results were obtained for each of the low 1, low 2 and low 3 ranges, these being represented here in Fig. 12(a) by the low 3 plot. The spectral-analysis results are shown in Fig. 12(b), indicating periodicities around 26 and 13.7 days. The medium 1 and low 3 displacements which are close to day zero are indicative of very short delays and are consistent with the 1 day delays found for GL-AGWs (see table 3 of Bowman and Shrestha, 1998). Also of significance were the results of similar $K_{P}$-sum and spectral analyses for low 3 levels for 1963-1966 and high levels for 1992-1997. 
NORMALIZED $K_{p}$ SUM (RM) VERSUS DARLING DOWNS RAINFALL
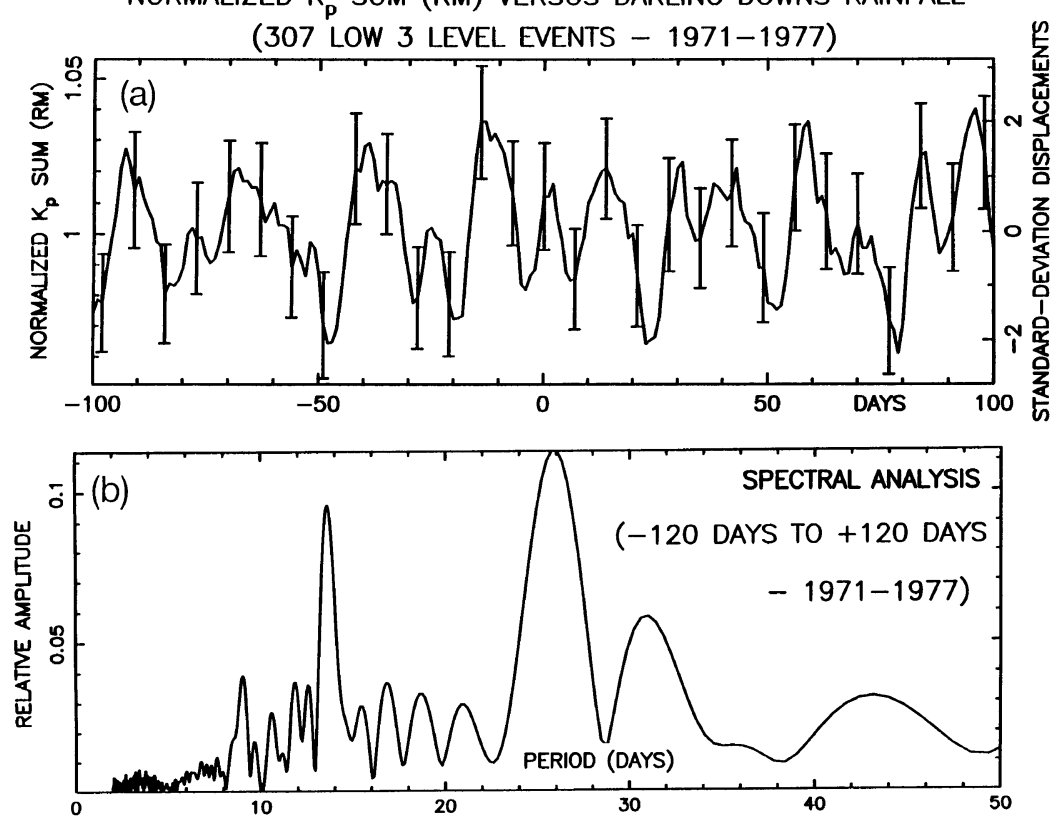

Fig. 12. (a) $K_{P}$ sum relative to Darling Downs low rainfall levels and (b) spectral analysis of Fig. 12(a) distribution.

\section{Discussion and Conclusions}

Over four sunspot-minimum periods, at reasonably-high levels of significance, consistent statistical results concerning relationships with EGA, involving delays of different magnitudes, have been found for a number of weather parameters ((a) GL-AGWs, (b) enhanced cold fronts, (c) rainfall areas in south-east Australia and (d) Darling Downs rainfall). Type 1 delays involve several days while type 2 delays are around 20 days. The EGA associated with these delays is part of sequences of recurrent geomagnetic activity involving periodicities around 27 days and 13.5 days. Looked at another way the $\Sigma K_{P}$ analyses have detected two 27-day periodicities which are displaced from each other by about 13.5 days, the 27-day periodicities recording about four or five cycles. Of the two sequences the one associated with the type 2 delays is significantly more dominant.

The 13.5 day periodicity in geomagnetic activity is now well documented (see Mursula and Zieger (1996) and references therein). Any discussion which related this recurrent geomagnetic activity to events on the sun must be considered speculative. However, as stated by Mursula and Zieger (1996) "Pap et al. (1990) suggest that the solar 13.5-day periodicity comes from two new magnetically active solar regions, about $180^{\circ}$ apart in longitude" (see also Donnelly and Puga, 1990). The observation by Mursula and Zieger (1996) that these periodicities last for a few (about 4) solar rotations is consistent with the results found here.

The experimental evidence indicates that certain weather parameters (e.g. GL-AGWs and enhanced cold fronts) are influenced by a mechanism of some kind resulting in delays of up to five days or more, following the occurrence of EGA (Bowman and Shrestha, 1998). These maxima (for these type 1 delays) in EGA are found by the present statistical analyses to form part of recurrent geomagnetic activity with a 13.5 day periodicity (Figs. 8(a) and 9(a)). This periodicity means that besides delays of about 5 days another maximum can be expected which indicates a delay in the vicinity of 20 days (type 2 delay). It is suggested that this explanation for the long delays may be sufficient without the need to find a mechanism. However there is a need of a mechanism to explain the type 1 delays. One hypothesis suggested and mentioned briefly by Bowman (2000) involves changes to weather systems, influenced by atmospheric gravity waves generated at times of delayed ionospheric D-region absorption. Another hypothesis considers the delays associated with the movements of weather patterns from polar regions following EGA (Bucha and Bucha, 1998).

Although weather parameters are used, the sole purpose of these analyses has been to establish the significance of associations they have with geomagnetic activity. Therefore the results are not concerned directly with meteorology, although they could help in the search for mechanisms to explain the relationships found.

Acknowledgments. The authors would like to thank the Brisbane Office of the Australian Bureau of Meteorology for providing the weather charts and rainfall information, and the World Data Center-C2 for Geomagnetism, Kyoto, Japan for making available the geomagnetic $\mathrm{H}$ components for a number of stations. The GLAGWs events for 1963-1966 were recorded by K. L. Shrestha as part of a PhD project.

\section{References}

Bowman, G. G., Atmospheric pressure waves at Brisbane and their association with certain ionospheric and solar events, Proceedings of AGARD Conference No. 115, 15-1, 1972.

Bowman, G. G., Associations between tropospheric gravity waves and enhanced geomagnetic over three sunspot-minimum periods, Indian $J$. of Radio Space Phys., 29, 1-8, 2000.

Bowman, G. G. and I. K. Mortimer, Quantitative estimates of relationships between geomagnetic activity and equatorial spread-F as determined by TID occurrence levels, Earth Planets Space, 52, 451-458, 2000.

Bowman, G. G. and K. L. Shrestha, Tropospheric atmospheric gravity 
waves and their relationship with geomagnetic activity, Indian J. Radio Space Phys., 27, 110-118, 1998.

Bucha, V. and V. Bucha, Jr., Geomagnetic forcing of changes in climate and in the atmospheric circulation, J. Atmos. Solar-Terr. Phys., 60, 145-169, 1998.

Croxton, F. E. and D. J. Cowden, Applied general statistics, 2nd ed., 749 pp., Pitman, London, 1955.

Donnelly, R. F. and L. C. Puga, Thirteen-day periodicity and the centerto-limb dependence of UV, EUV, and X-ray emission of solar activity, Solar Physics, 130, 369-390, 1990.

Duell, B. and G. Duell, The behavior of barometric pressure during and after solar invasions and solar ultraviolet invasions, Smithsonian Micell. Collections, 110(8), 1-34, 1948.

Mursula, K. and B. Zieger, The 13.5 day periodicity in the Sun, solar wind, and geomagnetic activity: The last three solar cycles, J. Geophys. Res., 101, 27,077-27,090, 1996.

Pap, J., W. K. Tobiska, and S. D. Bouwer, Periodicities of solar irradiance and solar activity indices, I., Solar Physics, 129, 165-189, 1990.

Roberts, W. O. and R. H. Olson, Geomagnetic storms and wintertime 300 $\mathrm{mb}$ trough development in the Pacific-North American area, J. Atmos. Sci., 30, 135-140, 1973.

Svensmark, H. and E. Friis-Christensen, Variation of cosmic ray flux and global cloud coverage - a missing link in solar-climate relationships, $J$ Atmos. Solar-Terr. Phys., 59, 1225-1232, 1997.

Taylor, J. R., M. Lester, and T. K. Yeoman, Seasonal variations in the occurrence of geomagnetic storms, Ann. Geophysicae, 14, 286-289, 1996.

Tinsley, B. A., Solar wind modulation of the global electric circuit and apparent effects on cloud microphysics, latent heat release, and tropospheric dynamics, J. Geomag. Geoelectr., 48, 165-175, 1996.

G. G. Bowman and I. K. Mortimer (e-mail: mortimer@physics.uq. edu.au) 